Marquette University

e-Publications@Marquette

Electrical and Computer Engineering Faculty

Research and Publications

Engineering, College of

$1-1-2011$

Application of Spatial Bandwidth Concepts to
MAS Pole Location for Dielectric Cylinders

James Richie

Marquette University, james.richie@marquette.edu

Accepted version. IEEE Transactions on Antennas and Propagation, Vol. 59, No. 12 (August 2011):

4861-4864. DOI.

(C) 2011 IEEE. Reprinted, with permission, from J. Richie, "Application of Spatial Bandwidth Concepts to MAS Pole Location for Dielectric Cylinders", IEEE Transactions on Antennas and Propagation, August 2011. 


\section{Application of Spatial Bandwidth Concepts to MAS Pole Location for Dielectric Cylinders}

James E. Richie, Senior Member, IEEE

\begin{abstract}
In this work, the concept of effective spatial bandwidth (EBW) is extended from the case of an MAS solution for perfectly conducting (PEC) cylinders to dielectric cylinders. It is shown that the ideas and results for the conducting cylinder apply in a straightforward manner to the dielectric case.

For the dielectric case, there are two auxiliary surfaces. Because the EBW calculations are independent of the scatterer material, the auxiliary surface for the scattered field will follow the same guidelines for both the PEC and dielectric cases. The guidelines for the second auxiliary surface are described and verified here. Guidelines for both a plane wave incident field and a monopole line source incident field are provided.
\end{abstract}

Index Terms-Electromagnetic scattering, boundary value problems

\section{INTRODUCTION}

The Generalized Multipole Technique (GMT) [1] and its variations can be used to compute the scattering from objects in a variety of scenarios. GMT and related methods compute the scattering from dielectric objects by placing canonical sources around the object. The scattered field is modeled using sources within the object, and the internal field is modeled using sources outside the object. Often, discrete multipoles are used for this purpose.

The Method of Auxiliary Sources (MAS) is one variation on the GMT family of techniques [2]. In MAS, the sources are placed on one or more auxiliary surfaces (AS). Fig. 1 shows a typical twodimensional scenario. For two dimensional scatterers, monopoles are placed on surface $\mathrm{AS}_{i}$ to model the scattered field; monopoles placed on $\mathrm{AS}_{o}$ model the internal field.

For $\mathrm{TM}^{z}$ two-dimensional problems, MAS defines a model for the scattered field:

$$
E_{z}^{s}(\vec{\rho})=\sum_{m=1}^{M_{o}} a_{m} H_{0}^{(2)}\left(k_{o}\left|\vec{\rho}-\vec{\rho}_{m}^{\prime}\right|\right)
$$

where $M_{o}$ is the number of poles (located on $\mathrm{AS}_{i}$ ); $k_{o}$ is the wavenumber outside the object; $a_{m}$ is the amplitude of pole $m$ (or the pole coefficient); and $H_{0}^{(2)}(\cdot)$ is the Hankel function of the second kind of order zero. The vector $\vec{\rho}_{m}^{\prime}$ is a vector from the origin to the location of pole $m$ and $\vec{\rho}$ points from the origin to some observation location on or outside the object.

For both the GMT and MAS methods, the best number and location for the canonical sources is often not clear. One approach to determine the number and location of the poles is to develop rules based on qualitative observations. In [3], an empirical scheme is provided for the location and number in two-dimensional problems based on a packing-number concept. In [4], appropriate GMT pole origins are determined using rule based algorithms. In all cases, it is well known that the auxiliary surface must enclose the singularities of the scattered field [1, Chapter 5] and [5]. The location and number of poles for GMT and related techniques are also discussed in [6], [7], and [2].

A second approach to determine the number and location of the poles is to investigate the convergence and accuracy of solutions using well-known scattering problems. The results of such investigations can then be applied to more general cases. Examples of such studies for GMT and MAS appear in [8], [9], and [10]. In [9], the MAS solution to normal incidence scattering by a PEC cylinder is

J. E. Richie is with the Department of Electrical and Computer Engineering, Marquette University, Milwaukee, WI.

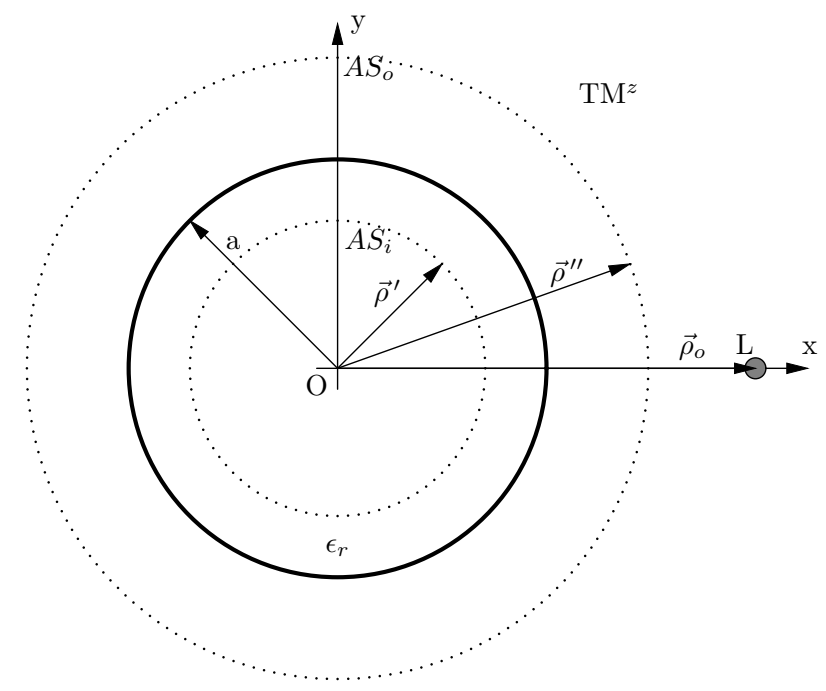

Fig. 1. Geometry of the two-dimensional scattering problem. Note the locations of $\mathrm{AS}_{i}$ and $\mathrm{AS}_{o}$ relative to the cylinder boundary at $\rho=a$.

investigated by analytically deriving and inverting the MAS matrix. Then, the condition number for the matrix is studied to ascertain the accuracy of the MAS solution. In [11], the methods of [9] are extended to the dielectric cylinder. In [12], a similar analytic treatment is performed on the MAS solution to oblique plane wave incidence on a dielectric cylinder.

Recently, the concept of effective spatial bandwidth (EBW) was applied to the scattering from conducting circular cylinders to develop pole placement guidelines [13]. By measuring the spatial bandwidth of the incident field on the surface of the scatterer, it is possible to develop insight toward suitable placement of poles in the MAS method. The results reported in [13] agree in principle with the conclusions drawn in [9].

This paper is an application of the tools and techniques developed in [13] for dielectric scatterers. For the dielectric case, the internal field is modeled as

$$
E_{z}^{d}(\vec{\rho})=\sum_{m=1}^{M_{o}} b_{m} H_{0}^{(2)}\left(k_{d}\left|\vec{\rho}-\vec{\rho}_{m}^{\prime \prime}\right|\right)
$$

where $k_{d}$ is the wavenumber within the object and $\vec{\rho}_{m}^{\prime \prime}$ is a vector from the origin to the location of pole $m$ on $\mathrm{AS}_{o}$, as shown in Fig. 1.

For the dielectric problem, the additional poles used to model the internal field must also have an effective spatial bandwidth that matches or exceeds the bandwidth of the field on the boundary.

The intention of this work is to add insight toward the effective implementation of MAS methods for more general problems by understanding the relationship between pole placement and spatial bandwidth of the fields. The concepts developed here can be easily extended to more general scatterer geometries.

\section{EFFECTIVE SPATIAL BANDWIDTH}

In this section, the method used to compute the effective spatial bandwidth of some field quantity along a boundary is explained. Then, a review of the results for the perfectly conducting cylinder will be provided.

As described in [13], a tangential field quantity $e(\phi)$ along a closed contour can be bandlimited to integer spatial frequency $N$ 
by performing the convolution

$$
e_{N}(\phi)=\frac{1}{C} \int_{0}^{2 \pi} e(\xi) B_{N}(\phi, \xi) \rho(\xi) d \xi
$$

where $e_{N}(\phi)$ is the bandlimited function, $C$ is the circumference of the scatterer boundary, $\rho(\phi)$ is the distance from the origin to the point on the scatterer at angle $\phi$, and $B_{N}(\phi, \xi)$ is

$$
B_{N}(\phi, \xi)=\frac{\sin \left[\left(N+\frac{1}{2}\right)(\phi-\xi)\right]}{\sin \left[\frac{1}{2}(\phi-\xi)\right]}
$$

The effective spatial bandwidth or EBW for $e(\phi)$ is defined as the smallest $N$ such that $\Delta_{N} \leq 0.1 \%$, where

$$
\Delta_{N}=\frac{\mathcal{E}(e)-\mathcal{E}\left(e_{N}\right)}{\mathcal{E}(e)} \times 100 \%
$$

where $\mathcal{E}(e)$ is the energy of $e$, given by

$$
\mathcal{E}(e)=\int_{0}^{2 \pi}|e(\phi)|^{2} \rho(\phi) d \phi
$$

The EBW for a field along a boundary is an integer due to the periodic nature of the field along the boundary.

Consider a perfectly conducting circular cylinder of radius $a$. The MAS model for the scattered field is given by (1). The auxiliary surface $\mathrm{AS}_{i}$ is a circle with radius $\rho^{\prime}<a$. The EBW for each pole is governed by the radius $\rho^{\prime}$.

If the incident field is a monopole line source at radius $\rho_{o}$, it is well known that $\rho^{\prime}>a^{2} / \rho_{o}$ will lead to acceptable results [8]. If the incident field is a plane wave, then $\rho^{\prime}$ is chosen so that the EBW for the MAS poles is equal to or larger than the incident field EBW. These results are demonstrated in [13].

The preceding analysis can be extended for a dielectric cylinder. This is due to the fact that the computation of EBW is independent of the composition of the cylinder. Values for EBW are the same for the perfectly conducting and dielectric cases.

\section{Method OF AuXILIARY SOURCES}

To solve the scattering problems, MAS shall be used. The scattered field is written as in (1) and the internal field is written as in (2). There is an incident field $E_{z}^{i}$ that scatters from the cylinder. The coefficients $\left(a_{m}\right.$ and $\left.b_{m}\right)$ are computed by satisfying the boundary conditions at $M_{o}$ points on the surface of the scatterer. Generally, 10 points per wavelength of circumference are more than sufficient for plane wave incidence; however, in the case of a monopole line source incident field, more matching points may be needed. There are $2 M_{o}$ unknowns, $M_{o}$ matching locations, and two boundary conditions at each matching location:

$$
E_{z}^{i}+E_{z}^{s}=E_{z}^{d} \quad \hat{n} \times\left[\vec{H}^{i}+\vec{H}^{s}\right]=\hat{n} \times \vec{H}^{d}
$$

Because the scatterer is a circular cylinder, the second boundary condition can be written as

$$
H_{\phi}^{i}+H_{\phi}^{s}=H_{\phi}^{d}
$$

where

$$
H_{\phi}=\frac{1}{j \omega \mu} \frac{\partial E_{z}}{\partial \rho}
$$

After some manipulations, the conditions are written as:

$$
E_{z}^{i}=\sum_{m=1}^{M_{o}}\left[-a_{m} H_{0}^{(2)}\left(k_{o}\left|\vec{\rho}-\vec{\rho}_{m}^{\prime}\right|\right)+b_{m} H_{0}^{(2)}\left(k_{d}\left|\vec{\rho}-\vec{\rho}_{m}^{\prime}\right|\right)\right]
$$

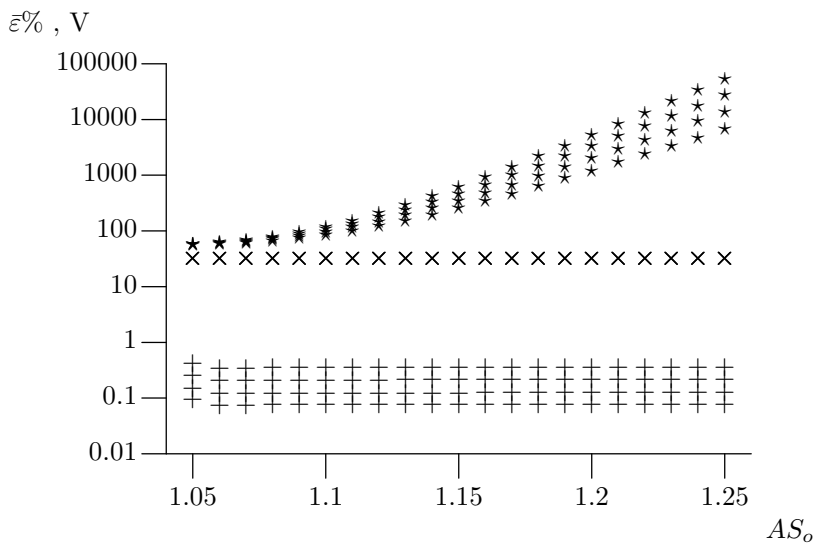

Fig. 2. Error (+) and measure values $V_{s}(\times), V_{d}(\star)$ for $a=1 \lambda, \rho_{o}=1.1 \lambda$, using fixed $A S_{i}=0.95 \lambda$ and varying $A S_{o}$. Results are shown for $M_{o}$ from 100 to 130 .

for the electric field, and

$$
H_{\phi}^{i}=\sum_{m=1}^{M_{o}}\left[-a_{m} H_{\phi, m}^{s}+b_{m} H_{\phi, m}^{d}\right]
$$

for the magnetic field. Eqns. (10) and (11) can be cast into matrix form $\mathbf{A} \mathbf{x}=\mathbf{B}$ where $\mathbf{A}$ is $2 M_{o} \times 2 M_{o}$, and $\mathbf{x}$ and $\mathbf{B}$ are vectors of length $2 M_{o}$. The vector $\mathbf{x}$ holds the unknown coefficients and the vector $\mathbf{B}$ has the known incident field values.

The quality of the solution is typically measured by finding the average boundary condition error (in percent) using:

$$
\bar{\varepsilon}_{E} \%=\frac{1}{360} \sum_{\phi=1}^{360^{\circ}} \frac{\left|E_{z}^{i}(\phi)+E_{z}^{s}(\phi)-E_{z}^{d}(\phi)\right|}{\left|E_{z}^{i}(\phi)\right|} \times 100 \%
$$

and

$$
\bar{\varepsilon}_{H} \%=\frac{1}{360} \sum_{\phi=1}^{360^{\circ}} \frac{\left|H_{\phi}^{i}(\phi)+H_{\phi}^{s}(\phi)-H_{\phi}^{d}(\phi)\right|}{\left|H_{\phi}^{i}(\phi)\right|} \times 100 \%
$$

The total error is computed using

$$
\bar{\varepsilon} \%=\sqrt{\left(\bar{\varepsilon}_{E} \%\right)^{2}+\left(\bar{\varepsilon}_{H} \%\right)^{2}}
$$

In [13], it was demonstrated that poor pole locations often result in very large coefficients with almost $180^{\circ}$ phase differences. A measure for the stability of the solution based on the coefficients $a_{m}$ was defined. This measure was shown to illustrate the suitability or numerical stability of the solution. For the scattered field poles, the measure $V_{s}$ is

$$
V_{s}=\frac{\left|a_{m}\right|_{\max }}{\left|\frac{1}{M_{o}} \sum_{m=1}^{M_{o}} a_{m}\right|}
$$

We shall define $V_{d}$ for the internal field poles similarly, replacing $a_{m}$ in (15) with $b_{m}$.

\section{RESULTS}

Consider the scattering of a monopole line source by a dielectric cylinder, as shown in Fig. 1. The cylinder has a relative permittivity of $\epsilon_{r}=4$ and a radius of $1 \lambda$. For $a=1 \lambda$, the spatial bandwidth of a monopole line source is equal to the plane wave bandwidth on the cylinder when $\rho_{o} \geq 1.3 \lambda$. Thus, we choose the monopole source to be at $\vec{\rho}_{o}$ along the $x$ axis $1.1 \lambda$ from the origin.

Fig. 2 shows the boundary condition error $\bar{\varepsilon} \%(+), V_{s}(\times)$ and $V_{d}(\star)$ for an auxiliary surface $A S_{i}$ at $0.95 \lambda$ and varying $A S_{o}$ from 


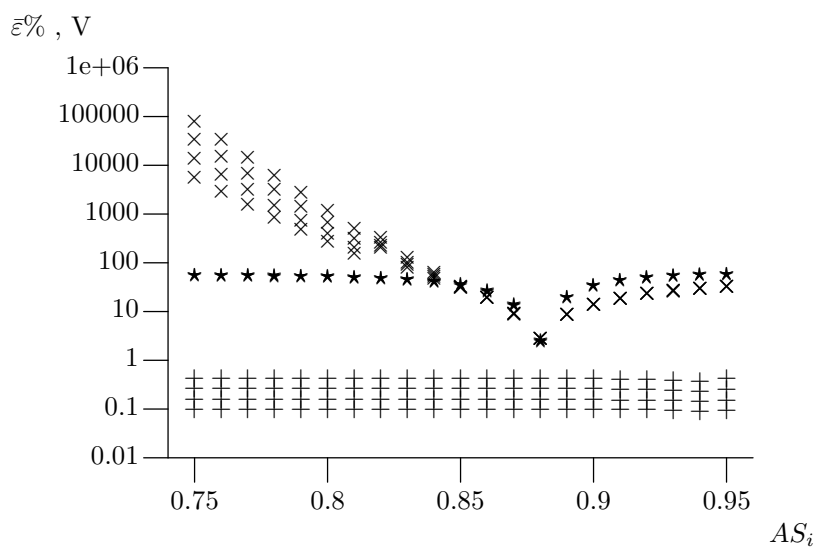

Fig. 3. Error(+) and measure values $V_{s}(\star), V_{d}(\star)$ for $a=1 \lambda, \rho_{o}=1.1 \lambda$, using fixed $A S_{o}=1.05 \lambda$ and varying $A S_{i}$. Results are shown for $M_{o}$ from 100 to 130 .

1.05 to $1.25 \lambda$. Each simulation was performed with $M_{o}$ from 100 to 130 in steps of 10 . The number of points is chosen quite large to illustrate stable error convergence in the results.

First consider $V_{s}(\times)$ in Fig. 2. Because the radius of $\mathrm{AS}_{i}$ is within the appropriate range (between $a$ and $a^{2} / \rho_{o}$ ), $V_{s}$ remains low as $\mathrm{AS}_{o}$ increases. In addition, as $M_{o}$ increases, $V_{s}$ remains constant. Note that each $\times$ symbol is actually four symbols on top of each other in the figure.

Next, consider the boundary condition error $\bar{\varepsilon} \%(+)$ in Fig. 2. As $\mathrm{AS}_{o}$ increases, the error remains fairly constant. As $M_{o}$ increases, $\bar{\varepsilon} \%$ decreases as illustrated by the vertical sets of + symbols. The behavior of $V_{s}$ and $\bar{\varepsilon} \%$ appears quite favorable.

Finally, consider $V_{d}(\star)$ in Fig. 2. For small values of $\mathrm{AS}_{o}$ (roughly less than $1.1 \lambda), V_{d}$ remains small and shows little variation with increasing $M_{o}$. However, as $\mathrm{AS}_{o}$ increases further, $V_{d}$ increases dramatically. In addition, as $M_{o}$ increases, $V_{d}$ also increases dramatically. This increase in $V_{d}$ indicates a less stable solution. Thus, the exterior poles should lie on an AS with radius between $a$ and $\rho_{o}$.

Fig. 3 shows $\bar{\varepsilon} \%, V_{s}$, and $V_{d}$ for an auxiliary surface $A S_{o}$ at $1.05 \lambda$ and varying $A S_{i}$ from 0.75 to $0.95 \lambda$. In this case, $V_{d}$ does not change with increasing $M_{o}$. The value of $\mathrm{AS}_{i}$ does not affect $V_{d}$ except near $A S_{i}=0.88 \lambda$. Again, the boundary condition error is consistent for the entire range of $\mathrm{AS}_{i}$ and decreases as $M_{o}$ increases. For $A S_{i}<0.85 \lambda, V_{s}$ increases greatly as $\mathrm{AS}_{i}$ decreases and as $M_{o}$ increases, illustrating the instability of the solution for these cases. The guideline that $\rho^{\prime}>a^{2} / \rho_{o}$ indicates that $\rho^{\prime}>0.909 \lambda$ and Fig. 3 indicates that $\rho^{\prime}$ should be roughly $0.85 \lambda$ or greater. Thus, the results are roughly consistent.

If both $A S_{i}$ and $A S_{o}$ are varied at the same time, the results shown in Fig. 4 are obtained. The results are plotted vs. $A S_{i} ; A S_{o}$ is found by taking $2 \lambda-A S_{i}=A S_{o}$, i.e., if $A S_{i}$ is $0.95 \lambda$, then $A S_{o}=2 \lambda-0.95 \lambda=1.05 \lambda$.

Fig. 4 shows that there is an optimum placement for the poles, near $A S_{i}=0.90 \lambda$. Not only is the error minimized, but the values of $V_{s}$ and $V_{d}$ are quite favorable.

The characteristics of the solution for the case of a plane wave incident field and increasing $\mathrm{AS}_{o}$ is now considered. Fig. 5 shows the results for $\bar{\varepsilon} \%$ and $V$ for a plane wave incident on a $1 \lambda$ cylinder with $\mathrm{AS}_{i}$ at $0.95 \lambda$. The radius of $\mathrm{AS}_{o}$ varies from 1.05 to $10 \lambda$. The range of $M_{o}$ for this data is also from 100 to 130 in steps of 10 .

First, note that the values for $V_{s}$ and $\bar{\varepsilon} \%$ are quite stable for all simulations.

For $\mathrm{AS}_{o}=1.05 \lambda, V_{d}$ is quite small indicating a stable solution. As $\mathrm{AS}_{o}$ increases to $5 \lambda$ and $10 \lambda, V_{d}$ increases, but not as dramatically

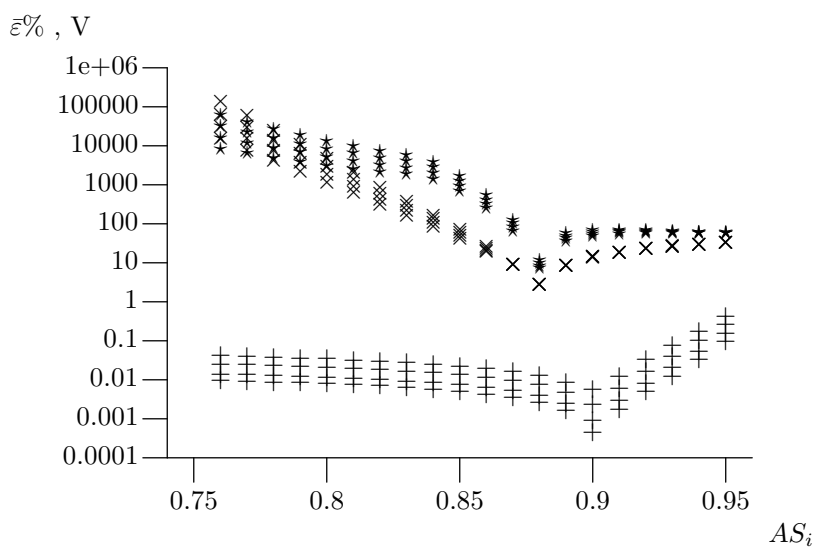

Fig. 4. Error(+) and measure values $V_{s}(\star), V_{d}(\star)$ for $a=1 \lambda, \rho_{o}=1.1 \lambda$, using variable $A S_{o}$ and $A S_{i}$ where $A S_{o}+A S_{i}=2 \lambda$. Results are shown for $M_{o}$ from 100 to 130 .

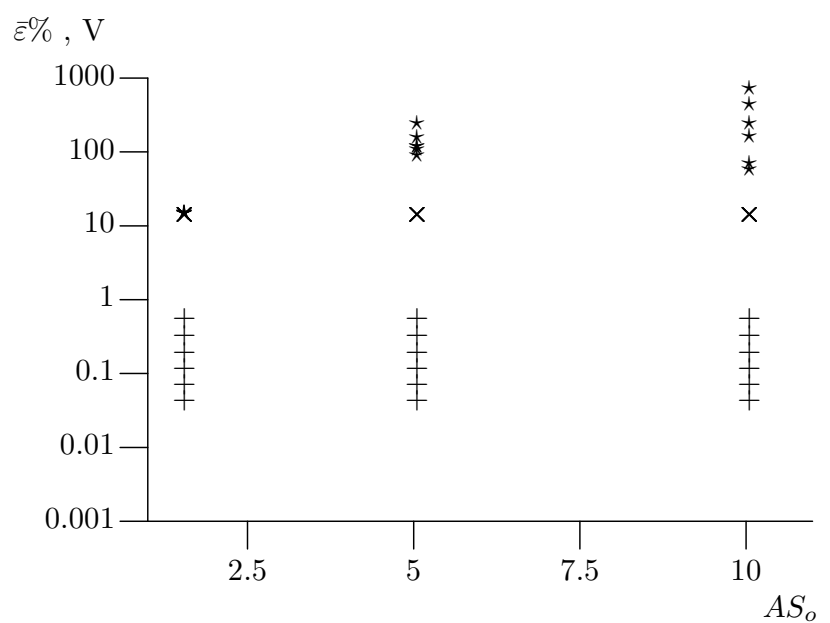

Fig. 5. Error(+) and measure values $V_{s}(\times), V_{d}(\star)$ for a plane wave incident on a $a=1 \lambda$ cylinder with $\mathrm{AS}_{i}$ fixed at $0.95 \lambda$ and various $\mathrm{AS}_{o}$. Note that $V_{d}$ does not increase dramatically with either increasing $M_{o}$ or increasing $\mathrm{AS}_{o}$.

as before (compare the vertical scale of Fig. 2 with Fig. 5).

Using a radius $\mathrm{AS}_{o}$ over $2.5 \lambda$ is not recommended; however, the results in Fig. 5 indicate that reasonably acceptable solutions are obtained even for very large $\mathrm{AS}_{o}$. Thus, the limit for $\mathrm{AS}_{o}$ with plane wave incidence is not clear and is not crucial to the solution.

\section{CONCLUSIONS}

In this work, the concept of effective spatial bandwidth has been extended from the perfectly conducting case [13] to dielectric cylinders. It has been found that the ideas and results for the conducting cylinder apply in a straightforward manner to the dielectric case.

Auxiliary surface locations for the dielectric case can be summarized as follows. For a monopole line source incident field at $\rho_{o}, \mathrm{AS}_{i}$ must be between $a^{2} / \rho_{o}$ and $a$; $\mathrm{AS}_{o}$ must be between $a$ and $\rho_{o}$. For a plane wave incident field, $\mathrm{AS}_{i}$ must be large enough for the monopoles to have a spatial bandwidth matching the plane wave variation; $\mathrm{AS}_{o}$ has no clear limit based on spatial bandwidth considerations.

It is expected that the insight developed in this work using spatial bandwidth concepts can be utilized to solve more general scattering problems. Using EBW as a guide for source placement shall lead to more stable solutions that also admit an acceptable boundary condition error. 


\section{REFERENCES}

[1] T. Wriedt, ed., Generalized Multipole Techniques for Electromagnetic and Light Scattering, vol. 4 of Computational Methods in Mechanics. New York, NY: Elsevier Science B. V., 1999.

[2] D. I. Kaklamani and H. T. Anastassiu, "Aspects of the method of auxiliary sources (MAS) in computational electromagnetics," IEEE Antennas and Propagation Magazine, vol. 44, pp. 48-64, June 2002.

[3] K. Beshir and J. Richie, "On the location and number of expansion centers for the generalized multipole technique," IEEE Trans. Electromagn. Compat., vol. 38, pp. 177-180, May 1996.

[4] E. Moreno, D. Erni, C. Hafner, and R. Vahldieck, "Multiple multipole method with automatic multipole setting applied to the simulation of surface plasmons in metallic nanostructures," J. Opt. Soc. Amer. A, vol. 19, pp. 101-111, Jan. 2002.

[5] Y. Leviatan, "Analytic continuation considerations when using generalized formulations for scattering problems," IEEE Trans. Antennas Propag., vol. 38, pp. 1259-1263, Aug. 1990.

[6] C. Hafner, The Generalized Multipole Technique for Computational Electromagnetics. Boston, MA: Artech House, 1990.

[7] P. B. Leuchtmann, "Automatic computation of optimum origins of the poles in the multiple multipole method (MMP method)," IEEE Trans. Magn., vol. M-19, pp. 2371-2374, Nov. 1983.

[8] G. Fikioris, "On two types of convergence in the method of auxiliary sources," IEEE Trans. Antennas Propag., vol. 54, pp. 2022-2033, July 2006.

[9] H. T. Anastassiu, D. G. Lymperopoulos, and D. I. Kaklamani, "Accuracy analysis and optimization of the method of auxiliary sources (MAS) for scattering by a circular cylinder," IEEE Trans. Antennas Propag., vol. 52, pp. 1541-1547, June 2004.

[10] H. T. Anastassiu, "Error estimation of the method of auxiliary sources (MAS) for scattering from an impedance circular cylinder," Progress in Electromagnetics Research, vol. PIER 52, pp. 109-128, 2005.

[11] H. T. Anastassiu and D. I. Kaklamani, "Error estimation and optimization of the method of auxiliary sources (MAS) for scattering from a dielectric circular cylinder," Radio Science, vol. 39, Oct. 2004. RS5015, DOI: 10.1029/2004RS003028.

[12] N. L. Tsitsas, E. G. Alivizatos, H. T. Anastassiu, and D. I. Kaklamani, "Optimization of the method of auxiliary sources (MAS) for oblique incidence scattering by an infinite dielectric cylinder," Archiv für Elektrotechnik (Electrical Engineering), vol. 89, pp. 353-361, 2007. DOI 10.1007/s00202-006-0019-1.

[13] J. Richie, "MAS pole location and effective spatial bandwidth of the scattered field," IEEE Trans. Antennas Propag., vol. 58, pp. 3610-3615, Nov. 2010. 